\title{
Prevalência de Obesidade e Fatores Potencialmente Causais em Adultos em Região do Sul do Brasil
}

\section{original}

\author{
Ediolane Hilbert Brati Vedana \\ Marco Aurelio Peres \\ JANAINA DAS NEVES \\ Gino Chaves da Rocha \\ GIANA Zabarto Longo
}

\section{Programa de Pós-graduação em Saúde Coletiva da \\ Universidade do Planalto Catarinense (Uniplac), Lages, $\mathrm{SC}$, Brasil (EHBV, GCR, GZL); Programa de Pós-graduação em Saúde Pública da \\ Universidade Federal de Santa \\ Catarina (UFSC), Florianópolis, \\ SC, Brasil (MAP); Departamento de Nutrição da UFSC, Florianópolis, SC, Brasil (JN).}

Recebido em 26/3/2008 Aceito em 11/8/2008

\author{
RESUMO
}

Objetivo: Estimar a prevalência de obesidade e os fatores potencialmente causais em adultos da região urbana de Lages, SC. Método: Estudo transversal de base populacional, realizado em adultos entre 20 a 59 anos $(n=2.002)$. A obesidade foi diagnosticada por meio do índice de massa corporal (IMC $\geq 30 \mathrm{~kg} / \mathrm{m}^{2}$ ). As variáveis independentes coletadas domiciliarmente por meio de entrevista foram idade, escolaridade, renda per capita, número de filhos, história familiar de obesidade e nível de atividade física. Foi realizada análise de regressão de Poisson, simples e múltipla. Resultados: A prevalência de obesidade foi de $23,5 \%$ (IC95\% 21,6-25,3), sendo maior em mulheres $(26,1 \%)$ do que em homens $(19,3 \%)$. No modelo final, ajustado por variáveis de confusão, mulheres mais idosas, de menor renda e com história familiar apresentaram maiores prevalências. Nos homens, a prevalência foi maior com o aumento da idade e entre os sedentários. Conclusão: Os fatores relacionados à obesidade são distintos entre os sexos. A prevalência da obesidade foi maior em mulheres e foi associada com menores rendas e idades mais avançadas. (Arq Bras Endocrinol Metab 2008; 52/7:1156-1162)

Descritores: Obesidade; Epidemiologia; Estudos populacionais em saúde pública; Prevalência

\section{ABSTRACT}

Prevalence of Obesity and Potential Causal Factors Among Adults in Southern Brazil.

Objectives: To estimate the prevalence of obesity and potential causal factors among adults living in the urban area of Lages, Southern Brazil. Methods: Population based cross-sectional study with a sample of 2,022 adults from 20 to 59 years-old. Obesity was assessed by the body mass index (BMI $\geq 30 \mathrm{~kg} / \mathrm{m}^{2}$ ). Exploratory variable were collected at adults' households using questionnaire and included age, schooling, per capita familial income, parity, obesity familial episodes, and level of physical activity. Simple and multiple Poisson regression analyses were performed. Results: The prevalence of obesity was 23.5 (95\% Cl 21.6-25.3). The prevalence of obesity was higher in women $(26.1 \%)$ than in men (19.3). In the final model, adjusted for confounders, were associated with obesity among women: older ages, low schooling and familial episodes of obesity; for men were associated with obesity: older ages and those with insufficient physical activity. Conclusion: Factors associated with obesity differ between men and women. The prevalence of obesity was higher in women and it was associated with low income and elderly. (Arq Bras Endocrinol Metab 2008; 52/7:1156-1162)

Keywords: Obesity; Epidemiology; Population studies in public health; Prevalence 


\section{INTRODUÇÃo}

A obesidade tornou-se preocupação mundial a partir de meados da década de 1990; desde então sua prevalência vem aumentando de maneira alarmante em praticamente todos os países. São apontados como causas a diminuição da atividade física e o maior consumo de alimentos pobres em nutrientes e em fibras e de alta densidade energética. Os riscos mais importantes da obesidade são o desenvolvimento de outras doenças crônicas não-transmissíveis (DCNTs), como diabetes tipo 2 e as doenças cardiovasculares, tendo como conseqüência maior risco de morte prematura ou redução da qualidade de vida do indivíduo (1).

Mais recentemente, segundo a Organização Mundial da Saúde (OMS), o sobrepeso atinge cerca de 1,6 bilhões de indivíduos acima de 15 anos, destes $400 \mathrm{mi}-$ lhões apresentam obesidade. A obesidade é responsável por cerca de $2 \%$ a $6 \%$ em média, do total de recursos financeiros destinados à saúde (2).

No Brasil, a região sul é a que possui as maiores prevalências de obesidade com índices semelhantes ou até mais elevados do que os encontrados nos países desenvolvidos (3).

Inquéritos nacionais, como o Estudo Nacional de Despesas Familiares (ENDEF), realizados entre 1974 e 1975 (4) e a Pesquisa sobre Padrões de Vida (PPV), realizada entre 1996 e 1997 (5), revelaram aumento da obesidade em todos os níveis de escolaridade para a população masculina. Para o sexo feminino, a relação entre a obesidade e a escolaridade foi semelhante à da população masculina no período de 1974/75 a 1989; entre 1974/75 e 1995/96 houve a manutenção da tendência de aumento apenas em mulheres de baixa escolaridade, apresentando estabilização ou, até mesmo, redução de obesidade entre mulheres de alta de escolaridade (6). Dados de 2003 da Pesquisa de Orçamento Familiar (POF) (7) revelaram que o excesso de peso afeta $41,1 \%$ dos homens e $40 \%$ das mulheres, visto que, desse grupo, a obesidade atingiu $8,9 \%$ dos homens e $13,1 \%$ das mulheres adultas.

São escassos os estudos de base populacional em cidades de médio porte, portanto, o presente estudo objetivou estimar a prevalência de obesidade e os fatores potencialmente causais no município de Lages, cidade de médio porte de Santa Catarina, sul do Brasil.

\section{MÉTODO}

O município de Lages situa-se na região serrana de Santa Catarina a $176,5 \mathrm{~km}$ da capital do estado, Florianópolis. A população do município no ano 2005 foi de 166.733 habitantes, sendo $97,4 \%$ na zona urbana (162.397 habitantes). No período de 1996 a 2000, a taxa anual de crescimento estimada foi de $1,4 \%$. O município apresenta razão de dependência de $53,8 \%$. A razão de dependência expressa a relação entre a população dependente economicamente (menores de 15 anos e idosos com 65 anos e mais) no que diz respeito à população economicamente ativa ( $15 \mathrm{a}$ 64 anos). O cálculo é feito da seguinte forma: soma-se a população de zero a 14 anos com a população com 65 anos e mais e divide-se o resultado pela população entre 15 e 65 anos.

$\mathrm{O}$ índice de desenvolvimento humano municipal

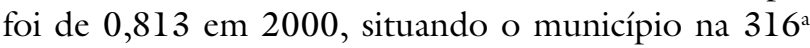
posição entre todos os municípios brasileiros e na $73^{\mathrm{a}}$ posição entre os municípios do estado de Santa Catarina. A mortalidade infantil foi de 22,8/1.000 nascidos vivos em 2004; a esperança de vida é de 71,9 anos; a taxa de fecundidade de 2,5 filhos por mulher; a média de anos de estudo foi de 6,6; e a taxa de analfabetismo foi de $8,4 \%$. A renda média per capita em 2000 foi de $\mathrm{R} \$ 335,40$ e a proporção de pobres foi de $22 \%$ (8).

Foi realizado inquérito epidemiológico de base populacional do tipo transversal. A população-alvo foi constituída de adultos de ambos os sexos, na faixa etária entre 20 e 59 anos de idade e residentes na zona urbana do município, totalizando cerca de $\mathbf{5 2} \%$ da população total ou 86.998 pessoas (9).

Este estudo é um subestudo de uma pesquisa mais abrangente cujo objetivo foi estudar a auto-avaliação das condições de saúde da população adulta de Lages e fatores associados (socioeconômicos, comportamentais, nutricionais, morbidades e utilização de serviços) (9). Para o cálculo da amostra do estudo mais amplo utilizou-se prevalência de $50 \%$ (desconhecida), com erro de 3,5 pontos percentuais, efeito do desenho de delineamento de $2 \%$, ao qual se somou $10 \%$ referente a perdas e recusas, mais $20 \%$ para o controle de fatores de confusão, calculado no módulo statcalc do pacote estatístico Epi Info (10), obtendo-se o total de 2.051 indivíduos. Para o cálculo do tamanho da amostra necessária para estimar a prevalência de obesidade considerou-se prevalência $(9,9 \%)$ encontrada em estudo realizado em Pelotas, RS (11), obtendo-se amostra 
mínima de 1.128 pessoas. O processo de amostragem foi realizado por meio de conglomerados, em dois estágios. Foram sorteados 60 setores censitários, cada um constituído por, aproximadamente, 300 domicílios (primeiro estágio) e 30 domićlios por setor (segundo estágio) (12). Para o início da pesquisa, em cada setor censitário foi sorteado um quarteirão e, neste, uma esquina (13). O número médio de moradores por domicílio equivale a quatro e, portanto, em cada um dos setores censitários existem, aproximadamente, 300 domicílios, o que equivale a 1.200 pessoas por setor censitário ou 720 pessoas $(60 \%)$ da faixa etária de interesse para a pesquisa. Como a amostra calculada para a pesquisa mais abrangente foi de 2.051 indivíduos, dividiu-se este valor pelo número de setores censitários sorteados (60 setores) e foi obtido o número aproximado de 34 pessoas por setor na faixa etária de 20 a 59 anos.

Foram considerados como perdas os residentes em domicílios visitados pelo menos quatro vezes, incluída, pelo menos, uma visita em finais de semana e outra noturna, sem que o entrevistador conseguisse localizar a pessoa a ser entrevistada ou caso houvesse recusa em participar. Foram critérios de exclusão as gestantes, os indivíduos amputados, os acamados, os portadores de aparelho gessado e os portadores de distúrbios psiquiátricos.

Para se obter a padronização no uso de critérios de medidas objetivas, como as medidas antropométricas, os observadores envolvidos no estudo participaram de treinamento prévio visando a capacitação da equipe. A padronização foi realizada com 30 adultos voluntários de ambos os sexos. Posteriormente à padronização, procedeu-se ao pré-teste do questionário, realizado também com 30 adultos da mesma faixa etária da pesquisa, em domicílios da área de abrangência de uma unidade de saúde do município. O instrumento utilizado para a coleta de dados foi um questionário com 186 perguntas, estruturado e prétestado, com o objetivo de obter informações sobre diferentes aspectos das condições de saúde da população adulta. A equipe de campo foi formada por 20 entrevistadores (dez duplas), todos os estudantes dos cursos da área de saúde da Universidade do Planalto Catarinense (Uniplac). O controle de qualidade dos dados foi efetuado pelo telefone, ao acaso, com aplicação de algumas questões do questionário em 10\% dos entrevistados.

\section{Variável dependente}

A variável desfecho foi o IMC, obtida por meio da razão do peso corporal em quilos pela altura em metros elevada ao quadrado $\left(\right.$ IMC $=$ peso/altura ${ }^{2}$ ). Para este estudo, a obesidade foi classificada como IMC $\geq 30$ $\mathrm{kg} / \mathrm{m}^{2}(2)$. O peso corporal e a altura foram aferidos de acordo com a técnica preconizada pela OMS. Para aferição do peso, foram utilizadas balanças digitais portáteis da marca Tanita com variação de $0,1 \mathrm{~kg}$ e capacidade de até $150 \mathrm{~kg}$; os participantes foram pesados uma única vez, e os valores registrados (14).

\section{Variáveis independentes}

Variáveis demográficas: Idade: em anos completos e categorizada em quatro grupos, quais sejam, 20-29, 30-39, 40-49 e 50-59 anos; Número de filhos (para as mulheres): nenhum, 1, 2 e 3 ou mais.

Variáveis socioeconômicas: Renda familiar: per capita em salários mínimos (equivalente a R \$380,00 ou, aproximadamente, 211 dólares norte-americanos), categorizada segundo os quartis da distribuição $(0,026-0,500 ; 0,510-0,880 ; 0,890-1,580 ; 1,590$ 19,740); Escolaridade em anos completos: 0-4, 5-8, 9-11 e 12 ou + anos; História familiar de obesidade: foi investigado se ambos os pais, ou pelo menos um deles, apresentavam obesidade.

\section{Variável comportamental}

Atividade física: o nível de atividade física foi categorizado como ativo e sedentário, segundo versão resumida em português do Questionário Internacional de Atividade Física (IPAQ) (15).

\section{Criação do banco de dados e análise estatística}

Após a criação do banco de dados, foi realizada dupla digitação dos dados no programa Epi Info, versão 6.0. A consistência e a análise dos dados foram realizadas no pacote estatístico Stata 9.0 (16).

Inicialmente foi realizada a análise descritiva. Para verificar as associações entre a variável dependente categórica (não-obesos $=0$ e obesos $=1$ ) e cada variável independente foi usado o teste qui-quadrado, conforme modelo hierárquico de determinação (Figura 1) (17). No modelo proposto, as variáveis socioeconômicas constituem o nível mais distal, as sociodemográficas 
e de história familiar de obesidade o nível intermediário e a variável comportamental, atividade física, a mais proximal ao desfecho.

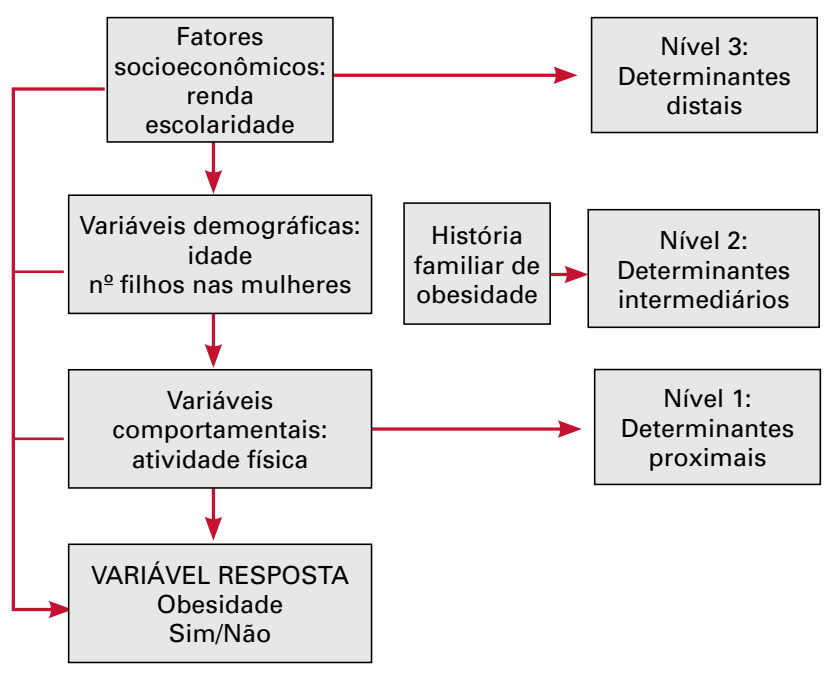

Figura 1. Modelo teórico de investigação dos efeitos de fatores relacionados ao sobrepeso e à obesidade estruturado em blocos hierarquizados.

A análise multivariável foi realizada por meio da regressão de Poisson, que apresenta como medida de efeito a razão de prevalência. A análise de regressão seguiu o modelo conceitual proposto (Figura 1).

As variáveis com $\mathrm{p}<0,25$ obtido na análise bivariada foram selecionadas para comporem a análise múltipla. $\mathrm{O}$ critério de permanência das variáveis nos seus níveis hierárquicos e no modelo final foi $\mathrm{p} \leq 0,05$ e/ou caso ajustassem o modelo. Foram apresentadas estimativas por ponto e por intervalo com $95 \%$ de confiança.

Todas as análises consideraram o efeito do delineamento do estudo usando-se o comando svy do Stata 9.0. O projeto do estudo foi submetido e aprovado pelo Comitê de Ética em Pesquisa da Uniplac sob Protocolo $\mathrm{n}^{\mathrm{O}}$ 01/2007. Foram solicitadas assinaturas dos termos de consentimento livre e esclarecido dos participantes da pesquisa. Caso fosse observado algum problema de saúde com o participante da pesquisa, o entrevistador o encaminhava à unidade de saúde mais próxima.

\section{RESULTADOS}

Entre os indivíduos investigados, 462 (23,5\%; IC95\%: $21,6-25,3)$ foram classificados como obesos. Observou-se maior prevalência de obesidade entre as mulheres, 314 (26,1\%; IC95\%: 23,6-28,6) do que entre os homens (19,3\%; IC95\%:16,5-22,1), motivo pelo qual foram realizadas análises estratificadas por sexo.

Nas análises bivariadas, encontrou-se, para o sexo masculino, relação linear positiva da obesidade com a idade $(\mathrm{p}<0,001)$ e negativa com a atividade física $(\mathrm{p}=$ $0,001)$. Entre as mulheres, também foi observada relação linear positiva da obesidade com a idade $(\mathrm{p}<$ $0,001)$, com a história familiar de obesidade $(\mathrm{p}<0,001)$ e com o maior número de filhos $(\mathrm{p}<0,001)$ (Tabela $\mathrm{l}$ ). Para renda e escolaridade, as associações foram negativas com valores de $p=0,001$ e $p<0,001$, respectivamente.

$\mathrm{Na}$ análise múltipla, foram associados à obesidade, em homens, o sedentarismo ( $\mathrm{RP}=1,55$; IC 95\% 1,21 $2,00)$ e o aumento da idade $R P=1,72$ para 30 a 39 anos e $\mathrm{RP}=2,15$ para 50 a 59 anos. Para o sexo feminino, observou-se que a obesidade aumenta com a idade, porquanto na faixa etária de 50-59 anos a RP é 2,62 (IC 95\%:1,88-3,65). Constatou-se, também, que a obesidade aumenta com a diminuição da renda e da escolaridade e com a história familiar de obesidade (Tabela 2).

\section{DISCUSSÃo}

A prevalência de obesidade encontrada na cidade de Lages foi de $23,5 \%$, superior em mais de três vezes a média mundial de 7\% (2); em nível nacional, as maiores prevalências são encontradas nas regiões sul e sudeste do Brasil (11). Em Lages, a obesidade apresenta-se maior entre as mulheres quando comparada aos homens $(26,1 \%$ e $19,3 \%$, respectivamente). Investigações com metodologias semelhantes realizadas na cidade de Pelotas, RS, nos anos de 1994 (1) e 2000 (12), mostram resultados similares ao presente estudo. Segundo recente inquérito nacional de fatores de risco para DCNTs do Instituto Nacional do Câncer, as prevalências de obesidade para a região sul variam de $12,2 \%$ a $10,9 \%(18)$.

Nesta pesquisa, a obesidade esteve positivamente associada com a faixa etária para ambos os sexos. $\mathrm{Na}$ população feminina, a prevalência de obesidade dobra 
Tabela 1. Prevalência de obesidade de acordo com variáveis exploratórias de acordo com o sexo (Lages, Santa Catarina, Brasil, 2007).

\begin{tabular}{|c|c|c|c|c|c|c|c|c|}
\hline & \multicolumn{4}{|c|}{ Masculino } & \multicolumn{4}{|c|}{ Feminino } \\
\hline & $n$ & $\begin{array}{c}\text { Prevalência de } \\
\text { obesidade } \\
\%\end{array}$ & $\begin{array}{l}\text { Razões de } \\
\text { prevalência }\end{array}$ & IC95\% & $\mathrm{n}$ & $\begin{array}{c}\text { Prevalência de } \\
\text { obesidade } \\
\%\end{array}$ & $\begin{array}{l}\text { Razōes de } \\
\text { prevalência }\end{array}$ & IC95\% \\
\hline \multicolumn{9}{|l|}{ Idade (anos) } \\
\hline $20-29$ & 257 & 11,30 & 1,00 & & 348 & 13,50 & 1,00 & \\
\hline $30-39$ & 172 & 18,68 & 1,63 & $1,04-2,56$ & 258 & 19,71 & 1,41 & $1,06-1,91$ \\
\hline $40-49$ & 181 & 24,99 & 2,12 & $1,39-3,24$ & 335 & 30,43 & 2,19 & $1,69-2,83$ \\
\hline $50-59$ & 155 & 27,14 & 2,30 & $1,42-3,71$ & 259 & 44,00 & 3,15 & $2,33-4,27$ \\
\hline \multicolumn{9}{|l|}{ Escolaridade (anos) } \\
\hline 12 e mais & 179 & 12,68 & 1,00 & & 269 & 17,55 & 1,00 & \\
\hline $9-11$ & 251 & 19,33 & 0,87 & $0,57-1,35$ & 340 & 20,62 & 1,16 & $0,83-1,63$ \\
\hline $5-8$ & 229 & 28,58 & 0,68 & $0,44-1,04$ & 328 & 28,11 & 1,54 & $1,03-2,33$ \\
\hline $0-4$ & 102 & 37,72 & 1,01 & $0,64-1,59$ & 244 & 40,64 & 2,27 & $1,69-3,04$ \\
\hline \multicolumn{9}{|l|}{$\begin{array}{l}\text { Renda familiar (per } \\
\text { capita - salário } \\
\text { mínimo) }\end{array}$} \\
\hline $1,59-19,74$ & 201 & 23,40 & 1,00 & & 256 & 18,44 & 1,00 & \\
\hline $0,89-1,58$ & 208 & 19,20 & 0,82 & $0,59-1,15$ & 295 & 26,82 & 1,39 & $0,99-1,95$ \\
\hline $0,60-0,88$ & 187 & 17,64 & 0,75 & $0,55-1,02$ & 299 & 26,13 & 1,39 & $0,96-2,00$ \\
\hline $0,026-0,59$ & 159 & 15,13 & 0,62 & $0,42-0,92$ & 329 & 31,33 & 1,65 & $1,18-2,33$ \\
\hline \multicolumn{9}{|l|}{$\begin{array}{l}\text { História familiar de } \\
\text { obesidade }\end{array}$} \\
\hline Não & 388 & 18,05 & 1,00 & & 539 & 19,32 & 1,00 & \\
\hline Pai ou mãe & 277 & 20,66 & 1,15 & $0,77-1,71$ & 467 & 29,56 & 1,50 & $1,21-1,87$ \\
\hline Ambos (pai e mãe) & 89 & 20,23 & 1,06 & $0,65-1,75$ & 167 & 38,37 & 2,00 & $1,54-2,60$ \\
\hline \multicolumn{9}{|l|}{ Número de filhos } \\
\hline nenhum & & & & & 228 & 16,25 & 1,00 & \\
\hline 1 & & & & & 269 & 17,87 & 1,06 & $0,74-1,55$ \\
\hline 2 & & & & & 304 & 27,60 & 1,64 & $1,21-2,23$ \\
\hline$\geq 3$ & & & & & 400 & 36,23 & 2,13 & $1,48-3,10$ \\
\hline \multicolumn{9}{|l|}{ Atividade física } \\
\hline Ativo & 290 & 21,74 & 1,0 & & 327 & 27,83 & 1,0 & \\
\hline Sedentário & 154 & 27,30 & 1,66 & $1,30-2,13$ & 826 & 25,36 & 1,18 & $0,89-1,40$ \\
\hline
\end{tabular}

após os 40 anos, em relação à faixa etária de 30 a 39 anos. Segundo dados do IBGE, homens e mulheres apresentam prevalências de obesidade semelhantes até os 40 anos, mas a partir desta idade, as mulheres apresentam prevalências duas vezes mais elevadas em relação aos homens (19).

A obesidade é determinada por vários fatores, visto que estes atuam em conjunto na determinação clínica da doença. Portanto, o resultado encontrado seria produto da combinação entre fatores genéticos, demográficos (maior idade), fatores ambientais e comportamentais (20).

As mulheres com maior escolaridade e com maior renda familiar apresentaram menor prevalência de obesidade, corroborando o estudo de Sichieri e cols. (21) e de Gigante e cols. (3) em Pelotas. Entre os homens, a associação entre obesidade e as variáveis socioeconômicas parece mais complexa. Na década de 1990, a obesidade passou a se deslocar para os estratos econômicos 
mais baixos (22). Foi demonstrado, a partir de 1997, que o risco de obesidade entre as mulheres na região sudeste e de áreas urbanas com baixa renda era cerca de duas vezes maior em relação a mulheres com maior renda $(5,6)$. Sichieri e cols. (21) afirmam que a evolução temporal da obesidade é predominante nas pessoas mais pobres, em ambos os sexos. Mediante análise da PNSN, realizada em 1989 (5), constatou-se que mesmo as famílias de menor renda convivem com o problema da obesidade, merecendo destaque às altas taxas de obesidade entre mulheres adultas pertencentes a famílias que vivem abaixo da linha de pobreza (6).

Estudo desenvolvido nacionalmente por inquérito telefônico em 2006 constatou que a prevalência de obesidade diminui no sexo feminino à medida que aumenta a escolaridade (23). A análise da relação entre obesidade e escolaridade, feita por Monteiro, Conde e Castro (24), mostrou que a prevalência de obesidade aumentou em todos os níveis de escolaridade para os homens nos anos de 1974 e 1989. Para as mulheres, houve mudança na prevalência de obesidade entre os períodos de 1974 a 1989. No primeiro período, a obesidade apresentava relação positiva com escolaridade, visto que no segundo período a obesidade era maior entre mulheres de menor escolaridade. A escolaridade representa a principal variável determinante da associação inversa encontrada no Brasil entre obesidade feminina e nível socioeconômico (24).

No que diz respeito à história familiar, observouse aumento de 2,5 vezes na prevalência de obesidade em indivíduos que referiram ter pai e mãe obesos. No estudo de Pelotas (3), observou-se que o risco de obesidade entre as pessoas que referiram ter pai e mãe obesos é de quase duas vezes maior do que aqueles cujos pais não apresentavam tal característica (3), possivelmente indicando a ação de fatores genéticos e ambientais e culturais.

Não obstante os pontos fortes desta pesquisa ressaltados anteriormente, é necessário salientar que, por se tratar de estudo transversal, não é possível estabelecer relação de causa e efeito entre as variáveis estudadas, uma vez que as informações sobre exposição e desfecho foram obtidas ao mesmo tempo. Outro fator limitante é a ausência de coleta de dados referentes à ingestão alimentar.

Existem algumas hipóteses para a explicação da ascensão da obesidade no mundo, entre elas a mudança nos padrões alimentares, observando-se diminuição no consumo (25) de fibras, frutas, hortaliças e os tradicionais alimentos da mesa brasileira, o arroz e o feijão,
Tabela 2. Associação entre obesidade e variáveis estudadas segundo o sexo. Razões de prevalência (RP), intervalos de confiança (IC95\%) e valores de p (Lages, SC, Brasil, 2007).

\begin{tabular}{|c|c|c|c|c|}
\hline \multirow[b]{2}{*}{ Idade (anos) } & \multicolumn{2}{|c|}{ Masculino } & \multicolumn{2}{|c|}{ Feminino } \\
\hline & $\begin{array}{l}\text { Razōes de } \\
\text { prevalência } \\
\text { (RP) }\end{array}$ & IC95\% & $\begin{array}{c}\text { Razōes de } \\
\text { prevalência } \\
\text { (RP) }\end{array}$ & IC95\% \\
\hline $20-29$ & 1,00 & & 1,00 & \\
\hline $30-39$ & 1,72 & $1,08-2,76$ & 1,14 & $0,82-1,58$ \\
\hline $40-49$ & 2,16 & $1,35-3,44$ & 1,72 & $1,26-2,34$ \\
\hline $50-59$ & 2,15 & $1,22-3,81$ & 2,34 & $1,64-3,34$ \\
\hline \multicolumn{5}{|l|}{$\begin{array}{l}\text { Escolaridade } \\
\text { (anos) }\end{array}$} \\
\hline 12 e mais & - & - & 1,00 & \\
\hline $9-11$ & & & 1,16 & $0,83-1,62$ \\
\hline $5-8$ & & & 1,32 & $0,89-1,95$ \\
\hline $0-4$ & & & 1,55 & $1,16-2,06$ \\
\hline \multicolumn{5}{|c|}{$\begin{array}{l}\text { História familiar } \\
\text { de obesidade }\end{array}$} \\
\hline Não & & & 1,00 & \\
\hline Pai ou mãe & - & - & 1,44 & $1,17-1,79$ \\
\hline $\begin{array}{l}\text { Ambos } \\
\text { (pai e mãe) }\end{array}$ & & & 2,17 & $1,72-2,75$ \\
\hline \multicolumn{5}{|c|}{ Atividade física } \\
\hline Sedentário & 1,00 & & & \\
\hline Ativo & 1,55 & $1,21-2,00$ & - & - \\
\hline
\end{tabular}

$(-)$ variáveis que não foram incluídas no modelo final, pois apresentaram $p>0,25$.

bem como do aumento (26) no consumo de produtos industrializados, ricos em gorduras, principalmente nas regiões mais desenvolvidas do Brasil (25), e açúcares, que conferem à dieta maior aporte calórico. Associa-se a esta hipótese a diminuição do gasto energético dos indivíduos em decorrência da urbanização da vida moderna (27).

Por meio de uma amostra representativa da população adulta de Lages, este estudo contribuiu para melhor entendimento sobre os fatores que se associam à obesidade em homens e mulheres, o que poderá subsidiar o planejamento de ações direcionadas à prevenção e ao manejo desta doença, levando-se em conta as diferenças demográficas, socioeconômicas e de estilo de vida observados.

Agradecimentos. Agradecemos à professora Izabella Barisson Matos, coordenadora do Programa de Pós-graduação em Saúde Coletiva da Uniplac, e à professora Karen Glazer Peres, coordenadora da pesquisa. Os autores declaram não haver conflitos de interesse científico neste artigo. 


\section{REFERÊNCIAS}

1. OPAS. Organização Pan-Americana da Saúde. Doenças crônico-degenerativas e obesidade: estratégia mundial sobre alimentação saudável, atividade física e saúde. Brasília, DF; 2003.

2. World Health Organization (WHO). Obesity: preventing and managing the global epidemic of obesity. Geneva: WHO; 2004.

3. Gigante DP, Barros FC, Post CLA, Olinto MTA. Prevalência de obesidade em adultos e seus fatores de risco. Rev Saude Publica. 1997;31: 236-46.

4. IBGE. Instituto Brasileiro de Geografia e Estatística [acesso em 2007 Mar 18]. Disponível em: http://www.ibge.gov.br.

5. Monteiro CA, editor. Velhos e Novos Males da Saúde no Brasil. São Paulo: Hucitec; 2000.

6. Monteiro CA, Conde WL. A tendência secular da obesidade segundo estratos sociais: nordeste e sudeste do Brasil, 19751989-1997. Arq Bras Endocrinol Metabol. 1999;43:186-94.

7. IBGE. Instituto Brasileiro de Geografia e Estatística. Pesquisa de orçamento familiar (POF), 2002/2003 [acesso em 2007 Mar 18]. Disponível em http://www.ibge.gov.br.

8. IBGE. Instituto Brasileiro de Geografia e Estatística. Contagem da população. Rio de Janeiro: IBGE; 2000.

9. Universidade do Planalto Catarinense (Uniplac). Programa de Pós-graduação em saúde coletiva. Auto-avaliação das condições de saúde e fatores associados: estudo de base populacional em adultos de Lages, Santa Catarina, 2007. Relatório final, Lages, SC, 2007. $51 \mathrm{p}$.

10. Dean AG, Dean JA, Colombier D, Brendel KA, Smith DC, Burton $\mathrm{AH}$, et al. Epi Info, version 6: a word processing, database, and statistics program for epidemiology on microcomputers. Atlanta, Georgia, USA: Centers for Disease Control and Prevention; 1994.

11. Gigante DP, Dias-da-Costa JS, Olinto MTA, Menezes AMB, Macedo S. Obesidade da população adulta de Pelotas, Rio Grande do Sul, Brasil, e associação com nível sócio-econômico. Cad Saude Publica. 2006;22:1873-9.

12. Bennett $S$, Woods $T$, Liyanage $W M$, Smith DL. A simplified general method for cluster-sample surveys of health in developing countries. WId HIth Statist Quart. 1991;44:98-106.

13. Barros FC, Victora CG. Epidemiologia da saúde infantil. Um manual para diagnósticos comunitários. São Paulo: Hucitec/ Unicef; 1998.

14. Lohman TG, Roche AE, Martorell R. Anthropometric standardization reference manual. Illinois: Human Kinetics Books; 1988.

15. Hallal PC, Dumith SC, Bastos JP, Reichert FF, Siqueira FV, Azevedo MR. Epidemiologia da atividade física no Brasil. Rev Saude Publica. 2007;41(3):453-60.
16. Stata Corp. Stata Estatical Software Release 9.0. Colege Station, Px: Stata Corporation; 2001.

17. Victora CG, Huttly SR, Fuchs SC, Olinto MTA. The role of conceptual frameworks in epidemiological analysis: a hierarchical approach. Int J Epidemiol. 1997;26:224-47.

18. Instituto Nacional do Câncer. Inquérito domiciliar sobre comportamento de risco e morbidade referida de doenças e agravos não transmissíveis [base de dados na Internet]. Rio de Janeiro: Inca, 2005 [acesso 2008 Feb 25, informações em publicação sobre o inquérito]. Disponível em: htpp://www.inca. gov.br/inquerito/sobrepesoobesidade.pdf.

19. IBGE. Instituto Brasileiro de Geografia e Estatística. Análise da disponibilidade domiciliar de alimentos e do estado nutricional no Brasil: pesquisa de orçamentos familiares 20022003. Rio de Janeiro; 2004.

20. Stunkard AJ. Factores determinantes de La obesidad: opinión actual. In: La obesidad en la pobreza: un novo reto para la salud pública. Washington DC: Organização Panamericana da Saúde. 2000;576:27-32.

21. Sichieri R, Coitinho DC, Pereira RA, Marins VMR, Moura AS. Variações temporais do estado nutricional e do consumo alimentar no Brasil. Rev Saude Coletiva. 1997;7:31-50.

22. Filho $M B$, Rissin $A$. A transição nutricional no Brasil: tendências regionais e temporais. Cad Saude Publica. 2003;19: 181-91.

23. Ministério da Saúde. Secretaria de Vigilância em Saúde. Disponível em http://portal.saude.gov.br/portal/arquivos/pdf/relatorio_vigitel_2006_marco_2007.pdf.

24. Monteiro CA, Conde WL, Castro IRR. A tendência cambiante da relação entre escolaridade e risco de obesidade no Brasil (1975-1997). Cad Saude Publica. 2003;19:S67-75.

25. Levy-Costa RB, Sichieri R, Pontes NS, Monteiro CA. Disponibilidade domiciliar de alimentos no Brasil: distribuição e evolução (1974-2003). Rev Saude Publica. 2005;39:530-40.

26. James WPT, Nelson M, Ralph A, Leather S. The contribution of nutrition to inequalities in health. BMJ. 1997;314:1545-9.

27. Gomes VB, Siqueira KS, Sichieri R. Atividade física em uma amostra probabilística da população do Município do Rio de Janeiro. Cad Saude Publica. 2001;17:969-76.

Endereço para correspondência:

Giana Zarbato Longo

Uniplac - Programa de Pós-graduação em Saúde Coletiva, Mestrado - Centro de Ciências Jurídicas

Av. Castelo Branco, 170 - Bairro Universitário

88.509-900 Lages, SC

E-mail: gianalongo@yahoo.com.br 